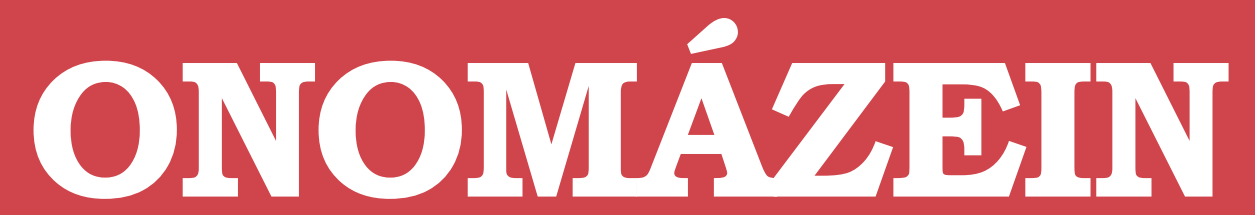

Revista de lingüística, filología y traducción
PONTIFICIA UNIVERSIDAD CATÓLICA DE CHILE FACULTAD DE LETRAS

\title{
“¿Cómo le gustaría que fuese la educación en lo referente a las lenguas?" Actitudes lingüísticas del alumnado mexicano*
}

"How would you prefer education to be regarding languages?" Mexican students' language attitudes

\author{
Isabel Corral Pérez \\ Instituto da Lingua Galega \\ Universidade de Santiago de Compostela \\ España
}

Número especial

Las lenguas amerindias en Iberoamérica: retos para el siglo XXI

2017
ONOMÁZEIN - Número especial

Las lenguas amerindias en Iberoamérica: retos para el siglo XXI (2017): 35-57

DOI: 10.7764/onomazein.amerindias.04

\section{(c) $($ i $)$}

Isabel Corral Pérez: Instituto da Lingua Galega, Universidade de Santiago de Compostela, España. | Correo electrónico: isabel.corral@usc.es 


\section{Resumen}

Esta investigación presenta los resultados de un estudio sobre las actitudes lingüísticas del alumnado universitario en México. Dicho trabajo se llevó a cabo mediante el diseño y la implementación de un cuestionario sociolingüístico que se aplicó a una muestra de estudiantes de la Universidad Autónoma Chapingo (UACh), ubicada en la ciudad de Texcoco de Mora en el estado de México. Se trata de una institución interesante por su orientación agrícola, lo que convierte a sus alumnos en informantes pertinentes, dado que no cuentan con formación sociolingüística específica, y por la gran diversidad étnica de sus alumnos.

El objetivo principal de este trabajo es conocer las actitudes lingüísticas del alumnado universitario mexicano hacia los hablantes de lenguas indígenas, incluidas las del alumnado con autoadscripción indígena hacia su endogrupo, y comprobar si existen diferencias destacables de acuerdo con la categoría étnica del informante.

Palabras clave: México; lenguas indígenas; actitudes lingüísticas; educación intercultural; derechos lingüísticos.

\section{Abstract}

This research presents the results of a study on language attitudes of Mexican university students. This work was carried out through the design and implementation of a sociolinguistic questionnaire, which was applied to a sample of students from the Autonomous University of Chapingo (UACh), located in the city of Texcoco de Mora in the state of Mexico. This is an interesting institution due to its agricultural orientation, what makes students relevant infor-

* Me gustaría agradecer a Modesto A. Rodríguez, M. Sol López y Carme Silva la lectura de versiones previas de este artículo y todas sus sugerencias. Igualmente a los revisores anónimos de Onomázein. Los errores que puedan persistir en el texto son de mi exclusiva responsabilidad. 
mants owing to their lack of specific sociolinguistic training, and because of the great ethnic diversity of the abovementioned university.

The main objective of this work is to explore Mexican students' language attitudes towards the indigenous language speakers, including the attitudes of the students that identify themselves as indigenous regarding their endogroup. We also want to verify if there are significant differences according to the ethnic category of the informant.

Keywords: Mexico; indigenous languages; language attitudes; intercultural education; linguistic rights. 
"[...] no tenemos conocimiento científico de ninguna característica lingüística que permita determinar si una lengua, dialecto, variedad lingüística o habla es mejor o peor [...] que otra, ya sea total o parcialmente" (Moreno Cabrera, 2000: 16).

\section{Introducción}

Lingüísticamente, México se caracteriza por la coexistencia entre el español y un total de 364 variantes ${ }^{1}$ lingüísticas articuladas en 68 agrupaciones y 11 familias. Dichas variantes se conciben como las unidades idiomáticas sujetas a los derechos establecidos por la Ley General de Derechos Lingüísticos de los Pueblos Indígenas (INALI, 2009), es decir, son las "lenguas indígenas nacionales" a las que se alude en esta disposición. Las lenguas originarias del país se encuentran en una situación de minorización con respecto al español -que funciona como lengua oficial de facto-, pese a la legislación que establece la misma validez y estatus². Este patrón de desequilibrio no es un caso aislado, sino característico de toda la región (Palacios, 2010).

Los factores que influyen en el mantenimiento o desaparición de las lenguas minorizadas son múltiples. A este respecto, se pueden consultar los trabajos de UNESCO (2003) y Palacios (2004), entre otros. La base de esta investigación es uno de estos factores, las actitudes lingüísticas, a las que nos aproximamos siguiendo el modelo de la triple composición de Rosenberg y Hovland (1960), según el que una actitud está constituida por tres elementos: cognitivo (pensamientos y creencias), afectivo (sentimientos) y conativo (disponibilidad para la acción). Estos elementos, aunque diferentes, comparten la disposición evaluativa frente al objeto de actitud (Ajzen, 2005 [1988]). Buscamos acceder tanto a las actitudes conscientes o explícitas como a las implícitas, si bien sabemos que sobre estas últimas existen menos posibilidades de control intencional y de verbalización (RAG, 2011: 33).

La tríada a la que hemos hecho referencia ha dado lugar al modelo mentalista multicomponencial. Dicho modelo se inserta dentro de la perspectiva mentalista, corriente teórica a la que nos adherimos, y que considera que las actitudes son un estado mental e interno del

1 Mantenemos la denominación utilizada por el INALI aunque no es la común en España, donde el término más frecuente es "variedad". En los demás casos empleamos este último.

2 El artículo 4 de la Ley General de Derechos Lingüísticos de los Pueblos Indígenas (2003) establece que "las lenguas indígenas que se reconozcan en los términos de la presente Ley y el español son lenguas nacionales por su origen histórico y tendrán la misma validez, garantizando en todo momento los derechos humanos a la no discriminación y acceso a la justicia de conformidad con la Constitución Política de los Estados Unidos Mexicanos y los tratados internacionales en la materia de los que el Estado Mexicano sea parte". 
individuo que predisponen su conducta al condicionar su respuesta ante un estímulo (Shuy, 1983, citado en Janés, 2006).

Las actitudes tienen dos motivaciones básicas, integradora o instrumental. La primera se relaciona con la pertenencia a un grupo, colectivo o comunidad, es decir, está vinculada a un componente social, mientras que la segunda es de corte individualista y se centra en cuestiones tales como la profesión, el estatus social, los logros personales o la mera supervivencia (Hernández Campoy, 2004). Partimos también de la convicción de que las actitudes no son fijas, sino que varían dependiendo de múltiples factores. Es por esto que se estudian teniendo en cuenta diferentes variables o determinantes. Baker (1992) habla de la edad, el género, la escuela, la competencia, los antecedentes lingüísticos y los culturales, si bien la lista es más amplia y puede incluir otros como el tipo de hábitat, la lengua inicial y habitual, la categoría de identificación étnica, etc. Siguiendo a Hernández Campoy (2004: 41), también consideramos que las actitudes van usualmente ligadas al factor prestigio, entendido como "un comportamiento lingüístico motivado por las actitudes sociales adoptadas ante determinadas formas lingüísticas [...]". El prestigio puede ser manifiesto o encubierto, según se exprese o no explícitamente, y no se refiere solo a la variación intralingüística, sino también a las lenguas per se y a sus hablantes.

Así pues, consideramos que el estudio de las actitudes es determinante en la lucha por el mantenimiento de la diversidad lingüística, puesto que, al influir en el uso que los hablantes hacen de las lenguas, también lo hacen en su pervivencia. Además, las actitudes intervienen en la planificación lingüística, que será diferente dependiendo de las actitudes del colectivo a quien se dirija (RAG, 2011). De acuerdo a esto, en el presente artículo se expondrán los resultados de un estudio actitudinal implementado en la Universidad Autónoma Chapingo (UACh).

\section{Objetivos y metodología de la investigación}

El objetivo principal de este estudio ${ }^{3}$ es conocer las actitudes lingüísticas del alumnado universitario mexicano hacia los hablantes de lenguas indígenas —en adelante LI-, incluidas las del alumnado con autoadscripción indígena hacia su endogrupo, y comprobar si existen diferencias destacables de acuerdo con la categoría étnica del informante. Asimismo, de forma secundaria, nos proponemos: 1) identificar sociolingüísticamente la muestra seleccionada; 2) presentar las lenguas originarias como elementos fundamentales de la identidad y la

3 La investigación que sustenta este trabajo fue realizada gracias a una ayuda de apoyo a la etapa predoctoral del Plan gallego de investigación, innovación y crecimiento 2011-2015 (Plan I2C), convocatoria 2013. 
cultura de los pueblos indígenas y romper con los estereotipos acerca de su supuesta escasa utilidad; 3) mostrar la importancia del ámbito educativo en los procesos de revitalización y mantenimiento de las lenguas indígenas, al ser el primer espacio de socialización y alfabetización de las niñas y niños fuera de la familia.

Desde el punto de vista metodológico, siguiendo el modelo multicomponencial de la corriente mentalista, partimos de la propuesta teórica de López Morales (2004 [1989]), quien establece una separación entre lo que creemos, la actitud manifestada ante lo que creemos y la manera en que actuamos. A partir de esta división, el lingüista considera que hay creencias de dos tipos: cognitivas y afectivas, mientras que las actitudes pueden ser bien positivas, bien negativas.

El estudio actitudinal que presentamos forma parte de un trabajo más amplio centrado en las actitudes lingüísticas del alumnado universitario mexicano, con el que comparte metodología y objetivos. Obviamente, el hecho de que aquel se trate de una investigación más amplia permite, además, realizar comparaciones interuniversitarias y confrontar la realidad sociolingüística de tres de las treinta y dos entidades federativas del país.

La parte central de esta investigación se basa en el análisis de los resultados de un cuestionario sociolingüístico diseñado para ser aplicado a alumnado universitario de tres universidades de México 4 , como parte del trabajo de campo desarrollado por la autora en el marco de una estancia de investigación realizada en la Universidad Autónoma Metropolitana - Unidad Iztapalapa entre septiembre de 2015 y enero de 2016.

Antes de la elaboración del cuestionario, se consultaron diversos instrumentos utilizados para medir las actitudes lingüísticas en diferentes estudios, entre ellos el empleado en el Mapa Sociolingüístico de Galicia 2004 (MSG04)5. Aunque sabemos que a priori ambas realidades lingüísticas presentan diferencias entre sí y requieren, por tanto, la creación de un instrumento a medida para su análisis, también somos conscientes de la existencia de rasgos comunes. Por ejemplo, tanto los informantes de este estudio como los del MSG04 proceden o viven en un contexto bilingüe o multilingüe, lo que hace que estén más predispuestos a tener una opinión

4 El cuestionario fue aplicado en tres universidades: Ia Universidad Autónoma Metropolitana - Unidad Iztapalapa (Ciudad de México), la Universidad Autónoma Chapingo (Texcoco de Mora, estado de México) y la Universidad Pedagógica Nacional - Unidad 201 (Oaxaca de Juárez, Oaxaca).

5 El Mapa Sociolingüístico de Galicia es un proyecto desarrollado por el Seminario de Sociolingüística de la Real Academia Galega con el objetivo de conocer la situación real de la lengua gallega para diseñar e implementar políticas lingüísticas adecuadas. El primer estudio se desarrolla en la década de los noventa y constituye el mayor trabajo de macrosociolingüística realizado hasta el momento en la Comunidad Autónoma de Galicia (España). En el año 2004 se hizo una actualización. 
o a posicionarse ante la diversidad lingüística, con independencia de que sean o no hablantes de una lengua minorizada. Además, estos dos trabajos presentan cuestiones que podemos considerar de interés universal, como la gestión de la diversidad en el ámbito educativo.

Con todo, es conveniente aclarar que el cuestionario utilizado está adaptado a la realidad sociolingüística y educativa mexicana y doblemente validado antes de su implementación: en primer lugar, fue sometido a una revisión por parte de varios expertos mexicanos, de la que se derivaron una serie de correcciones y mejoras que dieron lugar a un cuestionario piloto que fue testado en la UAM-I para detectar posibles problemas de forma o contenido. Tras esta aplicación experimental se introdujeron las correcciones pertinentes y se estableció la versión final.

La adopción del cuestionario como herramienta principal ${ }^{6}$ responde a sus ventajas con respecto a otros métodos, especialmente en cuanto permite obtener un mayor número de datos en un menor tiempo - la muestra total está compuesta por más de 450 informantes y los datos fueron recogidos en apenas 4 meses - y también porque facilita el procesamiento de las respuestas, así como la obtención de patrones, sobre todo si las preguntas son cerradas. Otro argumento a favor es el hecho de que este tipo de cuestionario se utiliza en estudios sociolingüísticos en todo el mundo.

El cuestionario empleado contiene 29 preguntas — solo cinco abiertas - estructuradas en tres bloques diferenciados: identificación social (4), identidad sociolingüística (16) y actitudes y creencias (9). Se contemplan un total de 65 variables. En este estudio presentamos los resultados del análisis de siete de ellas: cinco básicas, que nos permiten identificar sociolingüísticamente la muestra — “sexo”, "edad”, "categoría de autoidentificación étnica”, "lengua inicial" y "lengua habitual"-, y siete relacionadas con las actitudes y creencias. El soporte utilizado en este caso fue el papel. Los cuestionarios se repartieron en las aulas después de una breve presentación personal y del estudio en curso. El propio texto contenía las instrucciones para su cumplimentación. El proceso se presentó más como una ayuda por parte del alumnado a la investigadora —-doctoranda extranjera que se había desplazado a México con el objetivo de recoger datos - que como una observación en la que ellas/os eran el foco de estudio. En la despedida la investigadora agradeció la colaboración y se comprometió a dar a conocer los resultados, siguiendo el principio laboviano de la deuda contraída (González, 2008).

En cuanto al proceso de conteo, las respuestas — previamente codificadas - fueron introducidas en una base de datos del software de análisis estadístico SPSS, mediante el que se

6 En algunos casos, inmediatamente después de aplicar el cuestionario, se estableció un grupo de discusión en las aulas para debatir de manera informal y espontánea las principales cuestiones abordadas en el mismo. Además, también se realizaron entrevistas personales. 
realizó el cruce de las variables y se obtuvieron los resultados porcentuales que nos permitieron establecer los patrones actitudinales mayoritarios.

Nuestro campo de acción es la UACh, ubicada en el estado de México, en la parte centrosur del país. Es una institución con una clara orientación agrícola y donde ser indígena no es algo inusual7, de tal forma que podemos afirmar que Chapingo es un microcosmos diverso cultural, étnica y lingüísticamemente (Castillejos, 2011). Consideramos que se trata de un espacio ideal para el estudio de las actitudes lingüísticas, ya que, al ser un lugar de interacción entre diferentes culturas, es también caldo de cultivo para la aparición de fricciones entre las mismas y, en el caso que nos ocupa, de hipotéticos prejuicios, estereotipos y actitudes velada o abiertamente contrarias al mantenimiento de la diversidad cultural y lingüística mexicana.

En Chapingo convergen no solo mestizos e indígenas, sino también personas procedentes de diversos puntos del país ${ }^{8}$. Una parte importante del alumnado proviene de dos de los estados más empobrecidos`, lo que nos lleva a considerar otro de los rasgos de la institución: el bajo nivel socioeconómico de la mayor parte del estudiantado (Castillejos, 2011). Los datos recogidos pertenecen a una muestra de 97 informantes ${ }^{10}$, escogidos de manera aleatoria entre los 9115 matriculados (UACh, 2014). Contamos con un mostraje representativo que nos permite trabajar en un nivel de confianza del 95\%. Como ya se explicó anteriormente, los datos cuantitativos se complementan con la aplicación de instrumentos de tipo cualitativo, como los grupos de discusión y las entrevistas personales. En este artículo se presentan los resultados del análisis cuantitativo, que esperamos poder matizar en una segunda fase con el estudio del material complementario.

\section{Breve aproximación a la educación intercultural bilingüe (EIB)}

Hamel (1997) diferenciaba tres tipos de posturas ideológicas hacia los pueblos indígenas desde el momento de la invasión española de América hasta la actualidad: monoculturalismo,

7 Atendiendo a los datos del Anuario estadístico universitario 2012 (UACh, 2014), que solo recoge información acerca de la condición étnica del alumnado del nivel medio-superior y licenciatura, existe un $25,96 \%$ de matrícula indígena.

8 El primer estado de procedencia es Oaxaca (1114), seguido por la Ciudad de México (1109), Puebla (606), Chiapas (525) y Veracruz (455) (UACh, 2014).

9 De acuerdo a los últimos datos publicados por la versión mexicana de la revista Forbes, los diez estados más empobrecidos del país - que concentran el 81\% de la población en situación de pobreza- son, por orden, los siguientes: Chiapas, Oaxaca, Guerrero, Puebla, Michoacán, Estado de México, Guanajuato, Jalisco y Ciudad de México (Forbes Staff, 2015).

10 Querría expresar mi más sincero agradecimiento a todas las personas que participaron en este estudio. Sin su inestimable colaboración este trabajo no habría sido posible. 
multiculturalismo y pluriculturalismo. Pese a su composición plural, México —al igual que los demás países de la región - apostó desde el inicio de su vida independiente por la creación de un estado-nación a la europea (Comboni y Juárez, 2001), lo que supuso la homogeneización cultural y lingüística de la sociedad, hecho que educativamente implicó una continuación del modelo asimilacionista iniciado en época colonial.

Ya en las primeras décadas del siglo XX podemos hablar del inicio de una evolución del modelo educativo, al menos en el plano formal, que, poco a poco y con gran esfuerzo, se va desprendiendo del ideal castellanizante y empieza a ser consciente de la diversidad lingüística existente en las aulas y de la necesidad de gestionarla eficazmente en beneficio de la sociedad. Un impulso importante fue la recomendación de la UNESCO en 1951 acerca de la conveniencia de proporcionar educación en la lengua materna al menos en los primeros años de escolarización (Mar-Molinero, 2001).

En este contexto, el modelo de educación intercultural bilingüe surge en América Latina a comienzos de la década de 1980 como consecuencia del auge de los movimientos indígenas y de sus reivindicaciones, siendo uno de los componentes de la lucha político-social indígena y una de sus mayores conquistas (Williamson, 2004). Anteriormente, existieron experiencias educativas bilingües, pero con diferente objetivo; nos referimos a los sistemas basados en el bilingüismo de transición, que permitían la enseñanza y el uso de las lenguas indígenas como vía de acceso al ideal lingüístico perseguido: el monolingüismo en español. Por su parte, la EIB encaja en el modelo de bilingüismo de mantenimiento y desarrollo de ambas lenguas. Así, por EIB se entiende el modelo educativo que emplea la lengua y la cultura materna del educando para desarrollar su personalidad, abriéndose después a otras lenguas y culturas para complementarla (Albó, 2003). Se trata de un sistema con una doble dimensión: pedagógica, mediante la que incorpora en el proceso educativo las lenguas y culturas de los educandos procedentes de una minoría nacional, y política, que cuestiona el modelo educativo asimilacionista impuesto históricamente a los pueblos indígenas (Chiodi y Bahamondes, 2001).

Actualmente, en México está vigente la Ley General de Educación de $1993^{11}$ que reconoce el derecho de acceso de los hablantes de lenguas indígenas a la educación obligatoria en su propia lengua y en español (art. 7, apartado IV) ${ }^{12}$. Formalmente, se contempla la existencia de un sistema bilingüe, pero este no está funcionando en la práctica, al menos de forma gene-

11 El texto original fue publicado el martes 13 de julio de 1993 en el Diario Oficial de la Federación (DOF). La última reforma hasta la fecha es la publicada en el DOF el miércoles 1 de junio de 2016.

12 Este artículo establece también como uno de los fines de la educación impartida por el estado, sus organismos descentralizados y los particulares con autorización o reconocimiento de validez oficial de estudios "promover mediante la enseñanza el conocimiento de la pluralidad lingüística de la Nación y el respeto a los derechos lingüísticos de los pueblos indígenas" (apartado IV). 
ralizada (cfr. Jiménez y otros, 2015). La Ley General de Derechos Lingüísticos de los Pueblos Indígenas (2003) establece la igualdad de las lenguas indígenas y del español, todas ellas "lenguas nacionales" (art. 4) y y garantiza el acceso a una educación obligatoria bilingüe e intercultural, en los siguientes términos:

Las autoridades educativas federales y de las entidades federativas, [sic] garantizarán que la población indígena tenga acceso a la educación obligatoria, bilingüe e intercultural, y adoptarán las medidas necesarias para que en el sistema educativo se asegure el respeto a la dignidad e identidad de las personas, así como a la práctica y uso de su lengua indígena [...] (art. 11).

Pese a la legislación, la presencia de la LI en el aula es anecdótica y muchas veces se corresponde con los mecanismos del bilingüismo de transición (cfr. Reyes y Vásquez, 2008); otras veces se debe al compromiso y a los esfuerzos casi individuales de los docentes. En este último caso es importante tener en cuenta los problemas a los que se enfrentan: desubicación ${ }^{15}$, capacitación insuficiente, escasez de materiales en lengua indígena, falta de una variedad estándar, etc. (cfr. INEE, 2016).

\section{Análisis de los datos}

Escogimos la pregunta número 21 del cuestionario, reproducida en el título, como representante del bloque sobre actitudes. El motivo de esta elección es el hecho de que creemos que el educativo es un ámbito esencial tanto para el aprendizaje de las lenguas minorizadas como para el fomento de las actitudes lingüísticas positivas y la eliminación de los prejuicios lingüísticos. No obstante, y como ya hemos adelantado en el apartado acerca de la metodología empleada, en el estudio se tienen en cuenta más variables, además de las actitudinales propiamente dichas. En lo concerniente al análisis de las cinco variables “básicas”, se destaca lo siguiente.

13 La última reforma hasta el momento se publicó en el DOF el jueves 17 de diciembre de 2015.

14 "Las lenguas indígenas que se reconozcan en los términos de la presente Ley y el español son lenguas nacionales por su origen histórico y tendrán la misma validez [...]".

15 Es bastante común que los maestros de la EIB ejerzan en comunidades lingüísticas diferentes a la propia, lo que implica que la lengua originaria hablada por el docente y la del alumnado no es la misma. En los casos más leves se trata de variedades con un porcentaje alto de inteligibilidad, pero en otros hablamos de lenguas diferentes o de variedades muy alejadas. Esta situación de desubicación complica el funcionamiento de la educación bilingüe, puesto que, cuando las lenguas o variedades del profesor y de los alumnos no son inteligibles, inevitablemente la lengua vehicular es el español. Entendemos, por tanto, que una de las principales reivindicaciones de los maestros de educación bilingüe sea que la adjudicación del destino de trabajo tenga en cuenta la lengua. 
La distribución de edades es variada: Ia mínima recogida es de 14 años y la máxima de 26. La media se sitúa en los 18,54 años. Estamos ante un perfil de informantes jóvenes, que tradicionalmente son los que presentan actitudes más favorables ante las lenguas minorizadas; son quizá los más relacionados con la problemática lingüística y los más concienciados gracias, entre otros factores, a sus altos niveles de escolarización. Esta premisa se cumple sin ir más lejos en el caso gallego, al que se ha aludido, si bien los estudios de tipo cualitativo realizados en los últimos años para complementar e intentar dar explicación a algunos resultados del MSG han matizado dicha afirmación. Por lo que respecta al sexo, un 51\% de los informantes son hombres y un $49 \%$ mujeres, de tal forma que se obtiene una muestra bastante equilibrada y homogénea para la comparación.

Una de las variables que más interesa en nuestro estudio es la categoría de autoidentificación, es decir, el grupo étnico al que los propios informantes se adscriben. Las opciones prefijadas son: "afrodescendiente", "blanco", "indígena” y "mestizo", y los resultados obtenidos los que pueden observarse en el gráfico 1.

\section{GRÁFICO 1}

“¿Con qué categoría se autoidentifica?”

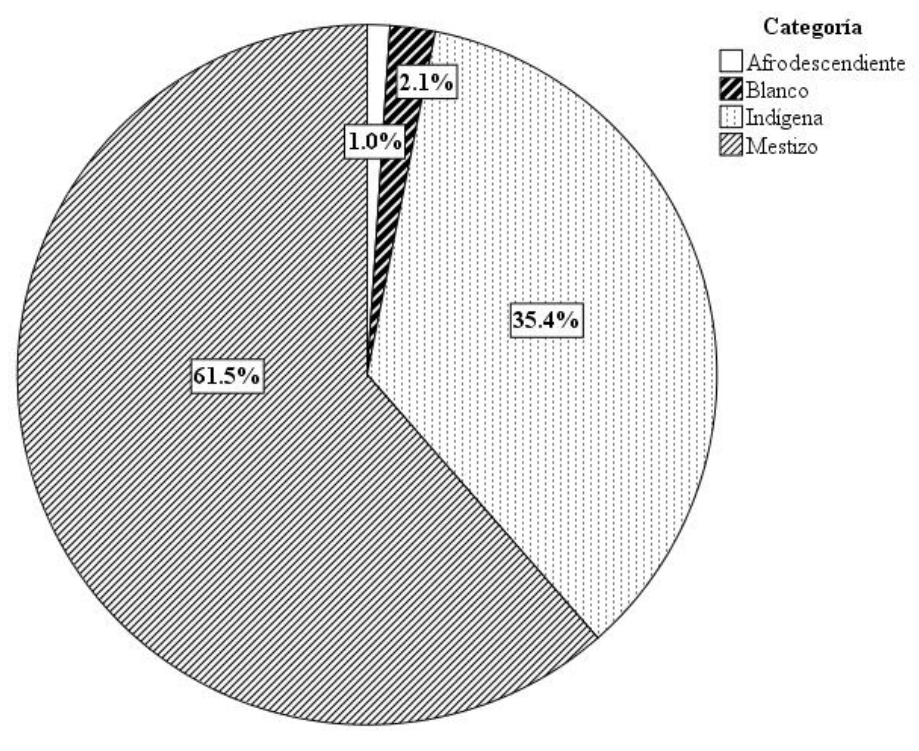

La categoría que cuenta con un mayor número de miembros es "mestizo", seguido de "indígena"; por otro lado, "blanco" y "afrodescendiente" son respuestas apenas representadas. Se trata de resultados previsibles que muestran el carácter mestizo del país y que enlazan con los últimos estudios censuales. De hecho, el Censo de Población y Vivienda (2010) determina que algo menos del 14\% de la población se considera indígena (INEGI, s. f., a), mientras que, según la Encuestra Intercensal 2015, hablamos del 21,5\% de la población total (INEGI, s. f., b). 
Otra de las variables básicas es la lengua inicial de los informantes. En este caso y, pese al porcentaje de alumnos que se identifican como indígenas, hay poca variación: el 81,9\% aprendió a hablar en español. Un 13,8\% es hablante inicial de LI y el 4,3\% se considera bilingüe inicial. El gráfico 2 presenta esta información de forma más visual, lo que nos permite apreciar la evolución en términos cuantitativos entre las opciones bilingües y monolingües en una lengua indígena y el monolingüismo inicial en español.

\section{GRÁFICO 2}

“¿En qué lengua aprendió a hablar?”

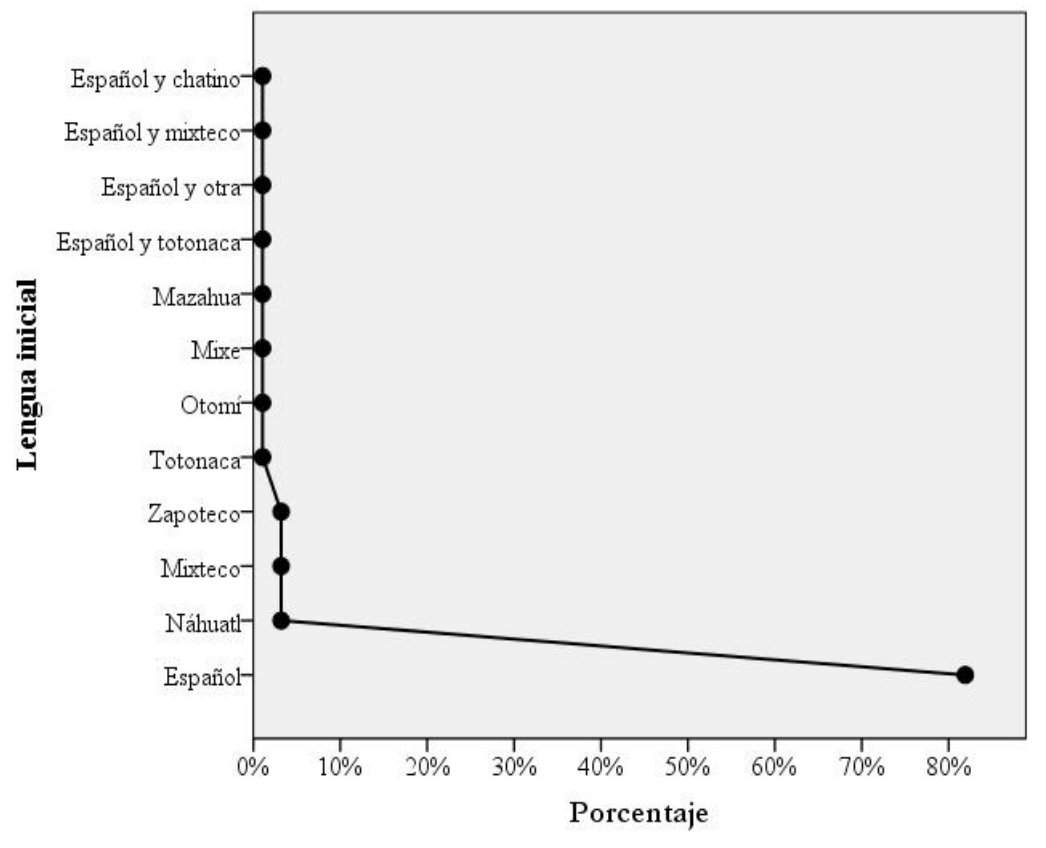

En lo concerniente a la lengua habitual, también hay un predominio absoluto del español (82,8\%). La opción bilingüe asciende en este caso al 13,8\% y la monolingüe en LI al 3,4\%. Estamos ante una cifra muy baja - más si tomamos en cuenta el porcentaje de autoidentificación-. Con todo, este desequilibrio puede estar motivado por los criterios externos sopesados en la identificación, ya que el lingüístico suele ser el criterio fundamental, pero no es el único. Los datos proporcionados por el cuestionario muestran que la lengua no es el rasgo más importante, pues solo lo considera así el 13,4\% de los informantes. En primer lugar, y con un $66,3 \%$ se sitúa el mantenimiento de las culturas, tradiciones, vestimenta, religiosidad, etc., propias de cada grupo étnico. El tercer rasgo es la autoidentificación (9,5\%). Destacamos también que un 4,2\% de los informantes afirmó que no existe ningún atributo que afecte en la identificación étnica de un individuo, lo cual puede interpretarse como un rechazo a la misma pregunta, esto es, como una oposición a la clasificación de las personas de acuerdo a su etnia, lo que puede esconder la existencia de actitudes negativas frente a lo indígena, 
un rechazo a una categorización que los ha marcado durante siglos. Entendemos que estas actitudes pueden ser implícitas, inaccesibles a la consciencia del informante y con escasas posibilidades, por tanto, de ser controladas o verbalizadas (RAG, 2011).

El dato más significativo, desde una perspectiva étnica, es la importancia que los mestizos conceden al uso de la LI (20,4\%), aspecto anecdótico para el grupo indígena (aprox. un 3\%). Es Ilamativo, sin embargo, que el criterio lingüístico sea más relevante para los no hablantes de una LI. En ambos casos la opción predominante es el mantenimiento de los rasgos culturales propios de cada pueblo (70,6\% en el grupo indígena y $66,1 \%$ en el mestizo). Otro dato curioso es que son los informantes mestizos los que consideran que no hay ninguna cualidad que defina o identifique a la población indígena como tal. Esta afirmación podría significar la inexistencia de atributos que funcionan como diferenciadores entre indígenas y mestizos, particularidades que sabemos que, en la práctica, sí que funcionan como tales, muchas veces de forma errónea ${ }^{16}$.

Volviendo a la variable "lengua habitual", tan solo cinco de los informantes afirman haber cambiado de lengua a lo largo de su vida. Cuando se les pregunta acerca del motivo, lo expresan con total claridad: por influencia de la educación. Ante tal afirmación y conociendo la tendencia castellanizadora que actúa en este ámbito, cabría esperar que estas cinco personas fuesen hablantes de LI hasta el cambio y que después pasaran a hablar español. Sin embargo, solo tres de los cinco informantes cumplen esta premisa, ya que tanto el hablante bilingüe como el de español pasaron a hablar otra lengua diferente a la habitual antes del cambio, pero no lo refirieron en el cuestionario. Por otra parte, el hecho de que el 94,7\% no haya cambiado de lengua a lo largo de su vida pone de relieve la importancia de la lengua inicial, que tiende a mantenerse, sobre todo si es la lengua dominante.

Si se tratan cuestiones más relacionadas con las actitudes y creencias lingüísticas, un aspecto importante atiende a la pregunta sobre la lengua que se les debe hablar a las niñas y los niños en casa, es decir, la que debe ser utilizada para la interacción en el hogar. Se trata de un tema crucial que marca las actitudes de los informantes ante la transmisión intergeneracional de la LI. Las opciones prefijadas en el cuestionario son: "español”; "español y lengua propia”; "solo en la lengua propia correspondiente”; "español, lengua propia y lengua extranjera”, e "inglés u otra lengua extranjera internacional”. Pueden contemplarse los resultados en el gráfico 3, que se reproduce seguidamente.

16 Nos referimos, por ejemplo, al hecho de identificar de forma equivocada a una persona como perteneciente a un pueblo indígena por tener determinados rasgos físicos asociados a este grupo en el imaginario colectivo. Si bien los atributos externos son los más extendidos, también existen estereotipos acerca de su carácter. 


\section{GRÁFICO 3}

"¿En qué lengua considera que se les debe hablar a las niñas/os en casa?"

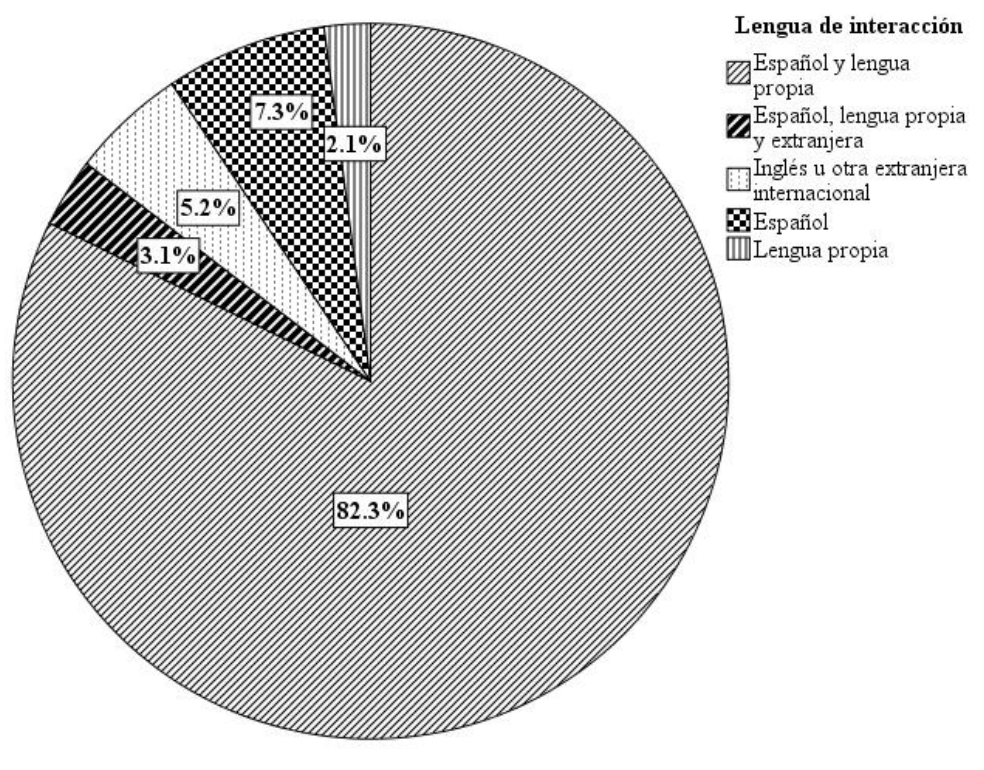

Tal y como puede observarse, predomina la opción bilingüe con un 82,3\%, elección que es, a nuestro juicio, la más enriquecedora, puesto que permite a niñas y niños mantener su lengua propia y participar a través de ella en la cultura y en la vida social de su comunidad y, al mismo tiempo, conocer y utilizar el español como instrumento de relación intercultural con el resto de la población, tanto de mestizos como de indígenas de otras etnias. El español sirve así de puente de acceso a otra cosmovisión y cultura de la que como mexicanos también forman parte. La segunda opción es la monolingüe en español (7,3\%), seguida, en tercer lugar, por el apoyo a las lenguas extranjeras con un 5,2\%. Tan solo un 2,1\% de los informantes defiende la transmisión de la LI como única. Este porcentaje es muy bajo, teniendo, además, en cuenta que se pregunta por el ámbito familiar, tradicionalmente reservado a la lengua materna, al ser un espacio en el que esta funciona como elemento de identificación grupal y que se caracteriza por un registro informal, no especializado y oral de la lengua. Creemos que la respuesta está influida por una visión instrumental de las lenguas, de acuerdo a la cual estas pueden proporcionar beneficios a corto o largo plazo, tales como reconocimiento social, ventajas económicas, etc. (Janés, 2006). La opción bilingüe es, además de la más beneficiosa, la más políticamente correcta. Desde una perspectiva cuantitativa, no tenemos forma de saber si detrás de esta respuesta se encuentra un deseo real de transmisión de las dos lenguas o cuáles son las causas de esta estrategia: visión positiva del bilingüismo, insuficiencia de las lenguas indígenas per se, etc.; no obstante, los grupos de discusión y los debates organizados apuntaron más en esta última dirección. Habría, consecuentemente, actitudes lingüísticas negativas y prejuicios implícitos. 
Llama también la atención la escasa diferencia entre el español como lengua única y la alternativa de las lenguas extranjeras. Sin duda, se trata de contestaciones mediadas por un criterio instrumental, lo que implica la existencia de actitudes más favorables ante las lenguas dominantes —especialmente frente a las internacionales - debido a las posibilidades laborales, económicas, de movilidad, etc., que ofrecen.

Respecto a la codificación de las respuestas atendiendo a las categorías de autoadscripción indígena o mestiza, el patrón de respuesta no se modifica sustancialmente, de tal forma que la opción predominante en ambos grupos sigue siendo la bilingüe, con porcentajes superiores al 80\%. La segunda opción es la trilingüe "español - lengua propia - lengua extranjera", seguida de la lengua extranjera como monolingüe, aunque son opciones sin apenas representación. Si se atiende a los resultados que presentan las lenguas indígenas en otros ámbitos, en el caso de los medios de comunicación la respuesta predominante tanto para los audiovisuales como para los escritos es la monolingüe en español, con porcentajes muy altos: 71,1\% y $81,4 \%$, respectivamente. Si se suman las opciones "bilingües" y "monolingües en LI”, estas aparecen en segundo lugar en ambos casos, con un $17,3 \%$ y un $12,2 \%$, respectivamente. Destacamos también las propuestas de traducción al inglés o incluso de monolingüismo en inglés, aunque son residuales. Desde una perspectiva actitudinal, interpretamos estos datos como negativos hacia las lenguas indígenas, al considerar superiores, ya sea en términos de prestigio o utilidad, las "grandes" lenguas de uso transnacional, como el español y el inglés. Una vez más, no existe un rechazo explícito a las lenguas originarias, pero sí un menosprecio velado.

La percepción de la superioridad de unas lenguas sobre otras está también presente en la pregunta sobre la lengua en que deberían estar escritos los libros de lectura obligatoria en las escuelas. A este respecto, despunta en primer lugar el español (72,1\%) y es reseñable el peso (9,3\%) de las opciones con preferencia por las lenguas extranjeras — sobre todo el inglés-, ya sea como forma única o con bilingüismo. Como dato anecdótico, cabe señalar que solo se alude explícitamente a dos lenguas indígenas: náhuatl ${ }^{17}(4,1 \%)$ y purépecha ${ }^{18}$ (1\%); las demás pierden su identidad bajo la etiqueta "indígenas".

17 Esta denominación genérica hace referencia a una de las 30 variedades lingüísticas diferenciadas englobadas dentro de la agrupación lingüística náhuatl, que, a su vez, pertenece a la familia yuconahua (INALI, 2009). De acuerdo al Atlas de los pueblos indígenas 2010 (Comisión Nacional para el Desarrollo de los Pueblos Indígenas, s. f.), los pueblos nahuas están localizados en los siguientes estados de México: Puebla, Veracruz, Hidalgo, San Luis Potosí, Guerrero, Estado de México, Ciudad de México, Tlaxcala, Morelos, Oaxaca, Tabasco, Tamaulipas, Michoacán, Jalisco, Durango y Nayarit.

18 La familia lingüística tarasca está integrada, en la actualidad, por un único idioma, el tarasco o purépecha - nombre en español de la autodenominación de la variedad - , que no ha podido ser relacionado genealógicamente con ningún otro. De esta forma, puede considerarse un idioma aislado. Se habla en el estado de Michoacán (INALI, 2009). 
La categoría étnica no resulta una variable relevante en este caso, al no aportar diferencias significativas: para los dos grupos la opción mayoritaria en los cuatro medios mencionados es la monolingüe en español, siendo los porcentajes ligeramente superiores en el grupo mestizo, excepto en el ítem referido a los libros de lectura obligatoria. Los porcentajes son superiores al $65 \%$ en todos los casos y grupos.

En cuanto a las actitudes que se pueden reflejar a través de los usos, seleccionamos cuatro colectivos de interlocutores: "familia”, "amigos", "profesores" y "doctor"; los dos primeros están relacionados con un uso privado e informal de la lengua, mientras que los otros dos remiten a ámbitos más formales y públicos. Si bien partíamos de la hipótesis de que las respuestas podrían mostrar una clara tendencia diglósica, es decir, un reparto de ámbitos y usos para cada lengua (Fishman, 1979), los resultados no son significativos a este respecto. No obstante, sí parecen igualmente alarmantes: muestran un evidente predominio del español como lengua de uso en los cuatro casos, aunque es cierto que los porcentajes son algo superiores en contextos formales. Los resultados son preocupantes porque evidencian una gran penetración del español en el hogar, baluarte de las lenguas minorizadas (UNESCO, 2003).

Las lenguas indígenas tienen escaso peso en los usos ligados a un registro formal. La elección del bilingüismo es mayor con familia (8\%) y amigos (6,1\%), inexistente con los profesores y anecdótica en la consulta del médico (1\%). Tanto indígenas como mestizos afirman utilizar predominantemente el español como lengua habitual con estos cuatro interlocutores. Se trata de una respuesta esperable para los grupos mestizo e indígena, cuando el receptor está vinculado a un ámbito formal y público, pero resulta chocante con la familia y los amigos. Se observa, pues, que la mayor presencia del español no depende de la identidad étnica del informante, sino del contexto: la utilización de lenguas originarias es inexistente con los profesores y casi con el médico, con un único caso.

Relacionada con los rasgos de identificación indígena vistos anteriormente, aparece la cuestión en la que se interroga acerca de la lengua/s9 que "debería" hablar una persona indígena. En este caso la opción bilingüe español-LI es la preeminente con un 93,8\%. La opción monolingüe en LI cuenta con un 5,2\%, mientras que solo un 1\% considera que el español es la única lengua que deben hablar. El gráfico 4 muestra esta información de forma más visual, al tiempo que proporciona los porcentajes exactos.

El bilingüismo se presenta, pues, como la opción más lógica. Estos datos refuerzan la idea de que la muestra encuestada no considera que el criterio lingüístico sea el más relevante

19 La pregunta es cerrada y las opciones de respuesta no contemplan como posibilidad la selección de lenguas extranjeras. 


\section{GRÁFICO 4}

"¿Qué lengua/s considera que debería hablar una persona indígena?"

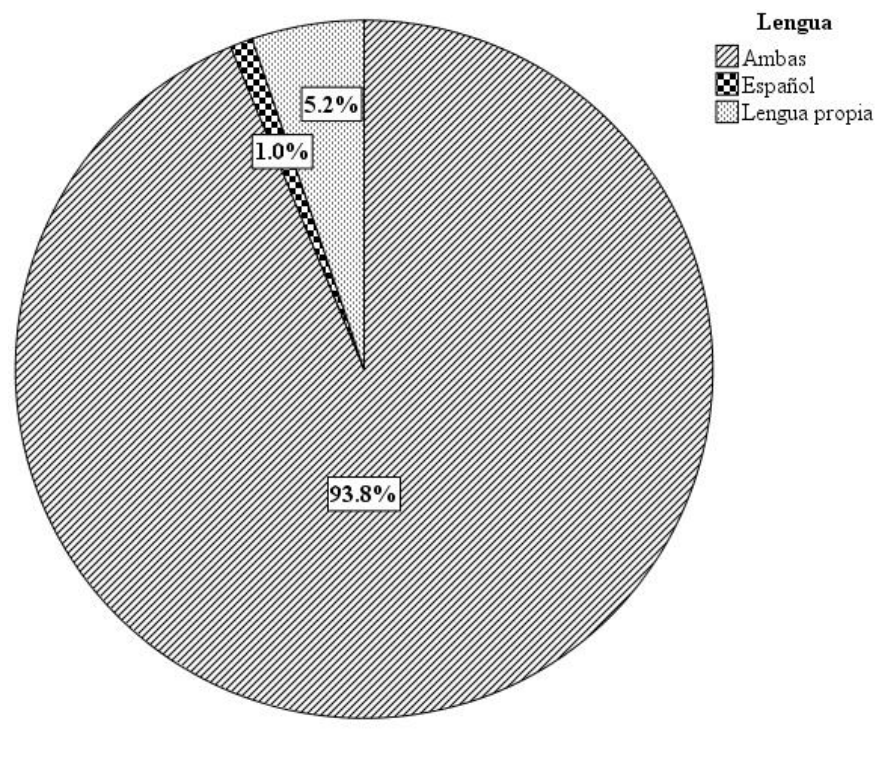

en la identificación étnica. Por lo que respecta a la interpretación actitudinal de esta respuesta, podemos considerar que se ha optado por la alternativa más neutral, aunque, como se comentó anteriormente, se desconocen los motivos que llevaron a esta elección. La autoadscripción étnica no afecta a estos resultados, de tal forma que la opción bilingüe continúa siendo la preferida. Por su parte, las alternativas monolingües suponen el 11,42\% en el grupo indígena y el 3,38\% en el mestizo. El monolingüismo en la LI tiene bastante más peso en el primer caso, obviamente.

Si se analiza el grado de conformidad de los informantes con una serie de afirmaciones que se les presentan sobre las lenguas indígenas, podemos establecer dos grupos: las relacionadas con la utilidad o proyección de estas y las vinculadas con aspectos identitarios y culturales. Las sentencias son las que se reproducen seguidamente: 1) "Son poco aptas para las necesidades del mundo moderno", 2) "Son menos útiles que las lenguas internacionales", 3) "No sirven fuera de su territorio", 4) "Identifican y diferencian a sus hablantes en un mundo global” y 5) "ayudan a mantener la tradición cultural de los pueblos que las hablan".

Los tres primeros casos presentan un patrón de respuesta idéntico: la opción mayoritaria es la más imparcial, es decir, la de los que afirman estar en parte de acuerdo y en parte en desacuerdo con la afirmación; y en los tres la suma de las opciones de rechazo —en mayor o menor grado - supera a la suma de las de apoyo. Por lo que respecta a los otros casos, la primera opción es la que avala el papel de la LI como símbolo identitario. En la número 4 casi la mitad de la muestra está totalmente de acuerdo con la afirmación, mientras que en la nú- 
mero 5 la cifra asciende al 84,5\%. Las respuestas de rechazo no alcanzan el 15\% en el primer caso y son mínimas en el segundo. Los resultados están dentro de lo esperado, ya que, aunque las actitudes ante las lenguas indígenas suelen ser negativas, es común que estas no se manifiesten abiertamente en instrumentos de este tipo: lo habitual son las respuestas políticamente correctas en las que el informante suaviza su opinión, intimidado por la presencia del entrevistador, al que se le presupone una opinión favorable ante las mismas. Esto explica que frecuentemente no exista correspondencia entre actitudes y usos reales ${ }^{20}$.

Nuevamente, los datos tampoco varían demasiado en función del grupo con el que se autoidentifique el informante. En cuatro de las cinco afirmaciones la opción destacada es la más neutral, como vimos en el análisis genérico. La excepción se da en una de las sentencias acerca del papel identitario de las lenguas indígenas, concretamente en la que afirma que los identifica y diferencia en un mundo global: la primera opción para ambos grupos es avalada con un $85,7 \%$ en el grupo indígena y un $83,1 \%$ en el mestizo. Rechazan más esta sentencia los indígenas (8,6\%) que los mestizos (5,1\%), dato llamativo, puesto que cabría esperar que fuesen los propios hablantes quienes más peso le concediesen a la lengua como símbolo de identidad, pero realmente vemos que en este caso la lengua está funcionando más como un elemento de diferenciación externa que de cohesión grupal21. Quizá esta respuesta muestre de forma encubierta una pequeña resistencia de los propios hablantes a ser considerados diferentes, es decir, que puede implicar prejuicios y actitudes negativas hacia el endogrupo, consecuencia quizá del rechazo y marginación de que han sido objeto durante siglos.

Por último, se presentan las respuestas a la pregunta que da título a esta investigación: “¿Cómo le gustaría que fuese la educación en lo referente a las lenguas?”. Como ya se ha adelantado, otorgamos especial relevancia a esta cuestión porque vincula las actitudes lingüísticas al grado de presencia de las lenguas originarias en el ámbito educativo, una cuestión prioritaria ya que "o ensino da lingua B implica sempre unha mudanza no prestixio da mesma [sic] a través da súa lexitimación polo emprego canónico que sempre implica o sistema educativo”22 (López, 2002: 49).

20 A este respecto, cabe insistir en que la no correspondencia entre actitudes y usos lingüísticos es una constante en muchos estudios actitudinales. Así ocurre, por ejemplo, en el caso gallego, según se desprende de los resultados del MSG04, al que ya aludimos anteriormente.

21 Una de las corrientes centrales de los estudios lingüísticos en la actualidad es la que surge a partir del binomio lengua e identidad y que enfatiza el valor de las lenguas como uno de los elementos identitarios más significativos, tanto a nivel individual como en la formación de la conciencia de grupo (König, 2001). Según estas teorías, para que una lengua sirva de base identitaria de una nación, debe cubrir dos necesidades: crear cohesión interna y diferenciación externa (Nadal, 2005).

22 "La enseñanza de la lengua B implica siempre un cambio en el prestigio de la misma a través de su legitimación por el uso canónico que siempre implica el sistema educativo”. La traducción es mía. 
La opción predominante en las respuestas obtenidas fue la monolingüe en español (36,6\%), seguida por la bilingüe equilibrada — 50\% de las materias en español y el otro 50\% en lengua indígena - con un 29\%. La tercera con mayor representatividad resultó ser el español como lengua vehiculary la LI solo como curricular (23,7\%). Si bien no se trata de un porcentaje muy alto, consideramos relevante que en este caso sí haya un predominio de la respuesta "Todas en español". Resulta interesante porque contrasta con las respuestas anteriores y deja al descubierto actitudes lingüísticas no tan positivas, sobre todo en lo referido a la visibilidad y/o utilidad de las lenguas indígenas. Si en la afirmación "Aprenderlas es una pérdida de tiempo" el 85,6\% dijo estar totalmente en desacuerdo, ahora tan solo el 1,1\% defiende que toda la docencia se imparta en la LI y el 3,2\% que sea toda en LI excepto la materia de español. Esto nos lleva a pensar que el apoyo realmente es hacia el aprendizaje de estas lenguas como materias curriculares, esto es, como si de lenguas extranjeras se tratase, y no una apuesta por el uso vehicular de las mismas como medios para transmitir otros aprendizajes.

\section{GRÁFICO 5}

“¿Cómo le gustaría que fuese la educación en lo referente a las lenguas?”

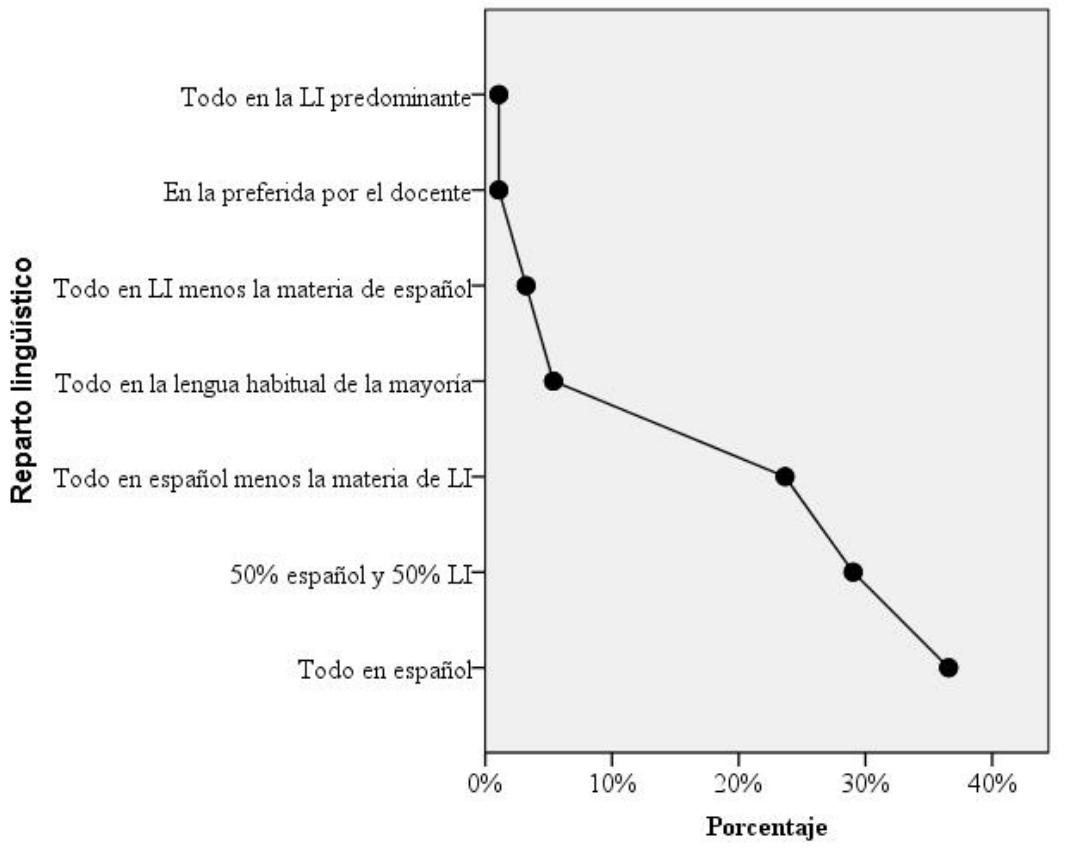

Los informantes también muestran predilección por el establecimiento de una norma lingüística prefijada, lo que se deriva del escaso peso de las opciones "Todo en la lengua habitual de la mayoría” (5,4\%) y “En la preferida por el docente” (1,1\%).

Las respuestas mediadas por la autoidentificación étnica muestran un predominio de la opción monolingüe en español, y del monolingüismo en español más la LI como curricular 
como segunda opción en el grupo mestizo. En el grupo indígena aparece la opción bilingüe en primer lugar, aunque, si sumamos las dos modalidades monolingües en español, tenemos una cifra superior. Podemos ver que la defensa de la presencia de las lenguas indígenas en el ámbito educativo - una de las principales reivindicaciones de los movimientos indígenas en toda la región - por parte de los informantes indígenas es mucho menor de lo esperado: tan solo un 5,7\% de los autoidentificados como indígenas defienden una educación monolingüe en una lengua originaria, mientras que los que contemplan su estudio únicamente como lengua curricular se sitúan en el 17,2\%. Tienen más peso los que apoyan una educación íntegramente en español (25,7\%). Para los mestizos, la opción monolingüe en LI es minoritaria (1,7\%).

\section{Conclusiones}

En primer lugar, cabe destacar la importancia del español como lengua inicial y habitual dentro de la muestra seleccionada. Conociendo el perfil de la UACh cabría esperar una mayor presencia de las lenguas indígenas, al menos como lengua inicial, pero este pequeño estudio nos permite dar cuenta de una preocupante pérdida de la transmisión intergeneracional de la LI, lo que también afecta directamente al número de hablantes habituales. De entre los dos tipos de argumentos para aprender una L2 —instrumentales e identitarios (Janés, 2006)—, los que influyen más son los relacionados con los beneficios que puede proporcionar dicho aprendizaje, de forma que los motivos para aprender una LI en la edad adulta serían escasos.

La principal conclusión alcanzada en este estudio es, pues, que las actitudes negativas hacia la LI y sus hablantes no se manifiestan explícitamente en Chapingo. Los resultados son bastante neutrales, lo que a priori induce a pensar que los prejuicios ya han sido superados. No obstante, si nos detenemos en el análisis, se observan pequeñas contradicciones que nos llevan a desconfiar de tal afirmación. Junto a la defensa abierta de las lenguas indígenas se produce también la de un sistema educativo monolingüe en español.

Pese a que predominan las respuestas que podemos considerar "imparciales" —creemos que mediadas por lo políticamente correcto-, también hay momentos en que los informantes respaldan el uso de las lenguas indígenas; no obstante, se trata, sobre todo, de cuestiones en las que se pregunta por vínculos identitarios y simbólicos, que se difuminan ante aspectos más concretos vinculados a la presencia real de la LI en ámbitos de uso público y formal.

Otro hallazgo interesante es el hecho de que en este estudio actitudinal la variable étnica no se muestra significativa: apenas hay diferencias importantes entre las respuestas de los informantes autoidentificados como indígenas y los mestizos. Así, no podemos afirmar que los prejuicios en un grupo pesen mucho más que en otro. Sin embargo, se advierte que los propios indígenas presentan actitudes negativas hacia su propia identidad etnolingüística. En relación con esta cuestión y como resultado no solo de esta investigación, sino también del contacto establecido 
durante la estancia con alumnado y profesorado indígena, estimamos que las actitudes negativas de los informantes indígenas van más allá de los argumentos tradicionales, basados sobre todo en la utilidad de las lenguas minoritarias, y se relacionan en mayor medida con la discriminación y el menosprecio sufrido por la población indígena durante siglos: es la negación en parte del endogrupo como consecuencia del rechazo sufrido por gran parte de la población mestiza.

\section{Bibliografía citada}

AJzen, Icek, 2005 [1988]: Attitudes, Personality and Behaviour, Poland: Open University Press.

Albó, Xavier, 2003: Cultura, interculturalidad, inculturación, Caracas: Federación Internacional de Fe y Alegría y Fundación Santa María.

BAKER, Colin, 1992: Attitudes and Language, Clevedon: Multilingual Matters.

Castillejos, Willelmira, 2011: Inseguridad lingüística de estudiantes universitarios. Estudio de caso de la Universidad Autónoma Chapingo. Tesis de doctorado, Universidad Autónoma Metropolitana - Unidad Iztapalapa [disponible en http://tesiuami.izt.uam.mx/uam/aspuam/presentatesis.php?recno=15617\&docs=UAMI15617.pdf, fecha de consulta: 10 de mayo de 2016].

Chiod, Francisco, y Miguel Bahamondes, 2001: Una escuela, diferentes culturas, Temuco (Chile, Corporación Nacional del Desarrollo Indígena): LOM Ediciones.

Combonı, Sonia, y José Manuel JuÁrez, 2001: "Educación, cultura y derechos indígenas: el caso de la reforma educativa boliviana”, Revista Iberoamericana de Educación 27, 125-154.

Comisión Nacional para el Desarrollo de los Pueblos indigenas (s. f.): Atlas de los pueblos indígenas 2010 [disponible en http://www.cdi.gob.mx/atlas/, fecha de consulta: 27 de octubre de 2016].

Congreso General de los Estados Unidos Mexicanos, 1993 [reformada]: “Ley General de Educación”, Diario Oficial de la Federación 13 de julio de 1993 [disponible en http://www.diputados.gob. mx/LeyesBiblio/pdf/137_010616.pdf, fecha de consulta: 30 de marzo de 2016].

Congreso General de los Estados Unidos Mexicanos, 2003 [reformada]: "Ley General de Derechos Lingüísticos de los Pueblos Indígenas”, Diario Oficial de la Federación 13 de marzo de 2003, [disponible en http://www.ordenjuridico.gob.mx/Documentos/Federal/html/w0108985.html, fecha de consulta: 31 de marzo de 2016].

Fishman, Joshua A., 1979: Sociología del lenguaje, Madrid: Ediciones Cátedra.

Forbes Staff, 23 de julio de 2015: "Los diez estados con más pobres en México" [disponible en http://www.forbes.com.mx/los-10-estados-con-mas-pobres-en-mexico/\#gs.vdbqox4, fecha de consulta: 4 de octubre de 2016]. 
González, Juan, 2008: "Metodología para el estudio de las actitudes lingüísticas" en Inés Olza, Manuel CaSAdo y Ramón GonZÁleZ (eds.): Actas del XXXVII Simposio Internacional de la Sociedad Española de Lingüística (SEL), Pamplona: Servicio de Publicaciones de la Universidad de Navarra, 229-238.

Hamel, Rainer Enrique, 1997: "Language conflict and language shift: a sociolinguistic framework for linguistic human rights", International Journal of the Sociology of Language 127, 105-135.

Hernández Campoy, Juan Manuel, 2004: “El fenómeno de las actitudes y su medición en sociolingüística”, Tonos Digital: Revista Electrónica de Estudios Filológicos 8, 29-56 [disponible en http://www.um.es/tonosdigital/znum8/portada/monotonos/04-JMCAMPOYa.pdf, fecha de consulta: 11 de noviembre de 2016].

Instituto Nacional para la Evaluación de la Educación (INEE), 2016: La educación obligatoria en México. Informe 2016 [disponible en http://publicaciones.inee.edu.mx/buscadorPub/P1///241/P1l241.pdf, fecha de consulta 10 de enero de 2017].

Instituto Nacional de Estadistica y Geografía (INEGI), (s. f., a): Censo de Población y Vivienda 2010 [base de datos], México: INEGI [disponible en https://www.cens02010.org.mx, fecha de consulta: 15 de abril de 2016].

Instituto Nacional de Estadistica y Geografia (INEGI), (s. f., b): Encuesta Intercensal 2015 [base de datos], México: INEGI [disponible en: http://www.inegi.org.mx/est/contenidos/Proyectos/encuestas/hogares/especiales/ei2015/, fecha de consulta: 15 de abril de 2016].

Instituto Nacional de Lenguas Indigenas (INALI), 2009: Catálogo de las lenguas indígenas nacionales. Variantes lingüísticas de México con sus autodenominaciones y referencias geoestadísticas, México: INALI.

Janés, Judit, 2006: "Las actitudes hacia las lenguas y el aprendizaje lingüístico", Revista Interuniversitaria de Formación del Profesorado 2 (20), 117-132.

Jiménez, Julián, y otros, 2015: "Análisis de las actividades de niños de Educación Inicial en pueblos originarios de Oaxaca, México”, Anthropologica 33 (35), 141-172.

KöNIG, Matthias, 2001: "La diversidad cultural y las políticas lingüísticas”, Gaceta de Derechos Humanos. Órgano informativo de la Comisión de Derechos Humanos del Estado de México 51, 86-92, [disponible en http://www.codhem.org.mx/localuser/codhem.org/info/gacetas/gaceta51.pdf, fecha de consulta: 1 de mayo de 2016].

López, Inmaculada, 2002: "A lingua galega e as leis. Notas para a reflexión”, Ianua. Revista Philologica Romanica 3, 43-61.

López Morales, Humberto, 2004 [1989]: Sociolingüística, Madrid: Gredos. 
Mar-Molinero, Clare, 2001: "Identidad nacional y educación bilingüe en el mundo hispano-hablante”, Revista de Educación 326, 79-97.

Moreno Cabrera, Juan Carlos, 2000: La dignidad e igualdad de las lenguas: crítica de la discriminación lingüística, Madrid: Alianza Editorial.

NADAL, Josep María, 2005: La llengua sobre el paper, Girona: CCG Edicions.

Palacios, Azucena, 2004: "Factores que influyen en el mantenimiento, substitución y extinción de las lenguas: Ias lenguas amerindias" en Ariadna LluIs I Vidal-Folch y Azucena Palacios (eds.): Lenguas vivas en América Latina, Barcelona-Madrid: Institut Català de Cooperaciò Iberoamericana - Universidad Autónoma de Madrid, 111-125.

Palacios, Azucena, 2010: "La lengua como instrumento de identidad y diferenciación: más allá de la influencia de las lenguas amerindias" en Rosa María Castañer y Vicente Lagüéns (coords.): De moneda nunca usada: Estudios dedicados a José Ma Enguita Urtilla, Zaragoza: Instituto Fernando El Católico / CSIC, 503-514.

Real Academia Galega (RAG) / Seminario de Sociolingüística, 2011: Actitudes lingüísticas en Galicia, A Coruña: Real Academia Galega, Seminario de Sociolingüística.

Reyes, Saúl, y Bulmano Vasquez, 2008: "Formar en la diversidad. El caso de la Escuela Normal Bilingüe e Intercultural de Oaxaca (ENBIO)", Trace. Travaux et Recherches dans les Amériques du Centre 53, 83-99 [disponible en http://www.redalyc.org/articulo.oa?id=423839509006, fecha de consulta: 10 de enero de 2017].

Rosenberg, Milton, y Carl Hovland, 1960: "Cognitive, Affective, and Behavioral Components of Attitudes" en Milton J. Rosenberg y otros (eds.): Attitude Organization and Change. An Analysis of Consistency Among Attitude Components, New Haven/London: Yale University Press, 1-14.

UNESCO, 2003: "Vitalidad y peligro de desaparición de las lenguas" [disponible en: http:// WwW.unesco.org/new/fileadmin/MULTIMEDIA/HQ/CLT/pdf/LVE_Spanish_EDITED\%20FOR\%20 PUBLICATION.pdf, fecha de consulta: 5 de abril de 2016].

Universidad Autónoma Chapingo (UACH), 2014: “Anuario estadístico universitario 2012" [disponible en: http://www.chapingo.mx/upom/descargas/ae/anuario_e_2012.pdf, fecha de consulta: 20 de abril de 2016].

Williamson, Guillermo, 2004: “¿Educación multicultural, educación intercultural bilingüe, educación indígena o educación intercultural?”, Cuadernos Interculturales 3 (2), 23-34. 